\title{
ON RECURRENCE COEFFICIENTS OF STEKLOV MEASURES
}

\author{
R. V. BESSONOV
}

\begin{abstract}
A measure $\mu$ on the unit circle $\mathbb{T}$ belongs to Steklov class $\mathcal{S}$ if its density $w$ with respect to the Lebesgue measure on $\mathbb{T}$ is strictly positive: $\inf _{\mathbb{T}} w>0$. Let $\mu, \mu_{-1}$ be measures on the unit circle $\mathbb{T}$ with real recurrence coefficients $\left\{\alpha_{k}\right\},\left\{-\alpha_{k}\right\}$, correspondingly. If $\mu \in \mathcal{S}$ and $\mu_{-1} \in \mathcal{S}$, then partial sums $s_{k}=\alpha_{0}+\ldots+\alpha_{k}$ satisfy the discrete Muckenhoupt condition $\sup _{n>\ell \geqslant 0}\left(\frac{1}{n-\ell} \sum_{k=\ell}^{n-1} e^{2 s_{k}}\right)\left(\frac{1}{n-\ell} \sum_{k=\ell}^{n-1} e^{-2 s_{k}}\right)<\infty$.
\end{abstract}

\section{INTRODUCTION}

Every probability measure $\mu$ on the unit circle $\mathbb{T}=\{z \in \mathbb{C}:|z|=1\}$ of the complex plane $\mathbb{C}$ generates the family of monic orthogonal polynomials $\Phi_{n}$ satisfying the recurrence relations

$$
\Phi_{n+1}(z)=z \Phi_{n}(z)-\bar{\alpha}_{n} \Phi_{n}^{*}(z), \quad z \in \mathbb{T}, \quad n \geqslant 0, \quad \Phi_{0} \equiv 1,
$$

where $\Phi_{n}^{*}$ are the "reversed" polynomials defined by $\Phi_{n}^{*}(z)=z^{n} \overline{\Phi_{n}(1 / \bar{z})}$. The recurrence coefficients $\alpha_{n}=-\overline{\Phi_{n+1}(0)}$ are completely determined by the measure $\mu$; in the non-trivial case where $\mu$ is supported on an infinite set, we have $\left|\alpha_{n}\right|<1$ for all $n \geqslant 0$. Any sequence of complex numbers $\alpha_{n}$ with $\left|\alpha_{n}\right|<1$ arises as the sequence of recurrence coefficients of a unique non-trivial probability measure on $\mathbb{T}$. A classical problem in the theory of orthogonal polynomials on the unit circle [9] is to relate properties of probability measures $\mu$ to properties of their recurrence coefficients $\left\{\alpha_{n}\right\}$.

In this paper we study recurrence coefficients of probability measures on $\mathbb{T}$ from Steklov class. Denote by $m$ the Lebesgue measure on $\mathbb{T}$ normalized by $m(\mathbb{T})=1$. A measure $\mu=w d m+\mu_{s}$ belongs to the Steklov class $\mathcal{S}$ if the density $w$ of its absolutely continuous part is strictly positive:

$$
\inf _{z \in \mathbb{T}} w(z)>0 .
$$

One version of famous Szegö theorem says that

$$
\prod_{k=0}^{\infty}\left(1-\left|\alpha_{k}\right|^{2}\right)=\exp \left(\int_{\mathbb{T}} \log w d m\right)
$$

for every probability measure $\mu=w d m+\mu_{s}$ on $\mathbb{T}$. If $\mu$ is a measure from Steklov class $\mathcal{S}$, then $\log w \in L^{1}(\mathbb{T})$, hence the product in the left hand side converges to a non-zero number and the recurrence coefficients of $\mu$ obey Szegö condition

2010 Mathematics Subject Classification. Primary 42C05, Secondary 33D45.

Key words and phrases. Orthogonal polynomials, Steklov conjecture, Muckenhoupt class, bounded mean oscillation.

The work is supported by RFBR grant mol_a_dk 16-31-60053 and by "Native towns", a social investment program of PJSC "Gazprom Neft". 
$\sum\left|\alpha_{k}\right|^{2}<\infty$. Another classical result, Baxter theorem, says that $\sum\left|\alpha_{k}\right|<\infty$ for the recurrence coefficients $\alpha_{k}$ of a probability measure $\mu$ on $\mathbb{T}$ if and only if $\mu$ is of the form $\mu=w d m$ for a strictly positive weight $w$ such that $\sum|\hat{w}(k)|<\infty$. Here and below $\hat{w}(k)=\int_{\mathbb{T}} w(z) \bar{z}^{k} d m(z), k \in \mathbb{Z}$, denote the moments of $w$. See Chapters 2, 5 in [9] for the proofs of Szegö and Baxter theorems. Summarizing, condition $\sum\left|\alpha_{k}\right|^{2}<\infty$ is necessary, while condition $\sum\left|\alpha_{k}\right|<\infty$ is sufficient for recurrence coefficients $\left\{\alpha_{k}\right\}$ to generate a measure $\mu \in \mathcal{S}$.

Further information on recurrence coefficients of Steklov measures could be extracted from Rahmanov example solved the classical Steklov problem. The original question by Steklov asks if a sequence of orthogonal polynomials $P_{n}$ on the interval $[-1,1]$ generated by a strictly positive weight $w$ on $[-1,1]$ is pointwise bounded:

$$
\sup _{n}\left|P_{n}(x)\right|<\infty, \quad x \in(-1,1) .
$$

This question and closely related issues attracted a lot of attention, see detailed review [10]. The negative answer was given by Rahmanov [7] in 1979. After transferring the problem to the unit circle, he constructed a strictly positive weight $w$ on $\mathbb{T}$ such that $\sup _{n \geqslant 0}\left|\Phi_{n}(1)\right|=\infty$ for the orthogonal polynomials $\Phi_{n}$ it generates. This weight $w$ can be chosen to be symmetric [8] with respect to the real line: $w(z)=w(\bar{z})$ for almost all $z \in \mathbb{T}$. Note that for every symmetric weight $w$ its orthogonal polynomials $\Phi_{n}$ satisfy $\Phi_{n}(1)=\Phi_{n}^{*}(1)$, hence

$$
\Phi_{n}(1)=\prod_{k=0}^{n-1}\left(1-\alpha_{k}\right), \quad n \geqslant 1 .
$$

This formula implies $\inf _{n \geqslant 0} s_{n}=-\infty$ for the partial sums $s_{n}=\alpha_{0}+\ldots+\alpha_{n}$ of recurrence coefficients $\left\{\alpha_{k}\right\}$ of the measure $w d m \in \mathcal{S}$ constructed in Rahmanov example. On the other hand, the Steklov bound

$$
\max _{z \in \mathbb{T}}\left|\Phi_{n}(z)\right| \leqslant \sum_{k=0}^{n}\left|\hat{\Phi}_{n}(k)\right| \leqslant\left\|\Phi_{n}\right\|_{L^{2}(\mathbb{T})} \sqrt{n+1} \leqslant\left(\inf _{\mathbb{T}} w\right)^{-1}\left\|\Phi_{n}\right\|_{L^{2}(\mu)} \sqrt{n+1},
$$

yields the estimate $s_{n} \geqslant-\frac{1}{2} \log n+c$ for all $n \geqslant 1$ and a constant $c$ independent of $n$ (to see this, use $\left\|\Phi_{n}\right\|_{L^{2}(\mu)} \leqslant 1$, formula (2), and the fact that $\sum\left|\alpha_{k}\right|^{2}<\infty$ ). Recent advances in the area show that the Steklov bound is optimal in a natural sense [1, 4. In particular, it follows from Theorem 4 in [1] and formula (2) that for every positive sequence $\left\{\varepsilon_{k}\right\}$ arbitrarily slowly tending to zero one can find a measure $\mu \in \mathcal{S}$ such that $s_{n_{k}} \leqslant-\frac{1}{2} \log \left(\varepsilon_{k} n_{k}\right)$ for some infinite increasing sequence $\left\{n_{k}\right\}$ of positive integers. See also [3], [5] for discussion of Rahmanov example and the corresponding recurrence coefficients.

In this paper we develop a method allowing to control oscillations of the sequence of partial sums, $\left\{s_{n}\right\}$, of recurrence coefficients of Steklov measures. Our main result is the following theorem.

Theorem 1. Let $\mu, \mu_{-1}$ be measures on the unit circle $\mathbb{T}$ with real recurrence coefficients $\left\{\alpha_{k}\right\},\left\{-\alpha_{k}\right\}$, correspondingly. If $\mu \in \mathcal{S}$ and $\mu_{-1} \in \mathcal{S}$, then

$$
\sup _{n>\ell \geqslant 0}\left(\frac{1}{n-\ell} \sum_{k=\ell}^{n-1} e^{2 s_{k}}\right)\left(\frac{1}{n-\ell} \sum_{k=\ell}^{n-1} e^{-2 s_{k}}\right)<\infty
$$

where $s_{k}=\alpha_{0}+\ldots+\alpha_{k}$ for integer $k \geqslant 0$. 
The Muckenhoupt class $A_{2}(\mathbb{R})$ on the real line $\mathbb{R}$ consists of functions $g$ such that

$$
\sup _{I \subset \mathbb{R}}\left(\frac{1}{|I|} \int_{I} g(t) d t\right)\left(\frac{1}{|I|} \int_{I} \frac{1}{g(t)} d t\right)<\infty,
$$

where the supremum is taken over all intervals $I \subset \mathbb{R}$. A similarity between relations (44) and (5) explains the name "discrete Muckenhoupt condition" we use for referring to (4). One may observe that in the setting of the Baxter theorem the partial sums $s_{k}$ are uniformly bounded and hence relation (4) is obviously satisfied. Jensen's inequality implies that the sequence $s=\left\{s_{k}\right\}$ in Theorem 1 has bounded mean oscillation:

$$
\sup _{n>\ell \geqslant 0} \frac{1}{n-\ell} \sum_{k=\ell}^{n-1}\left|s_{k}-\langle s\rangle_{n, \ell}\right|<\infty, \quad\langle s\rangle_{n, \ell}=\frac{1}{n-\ell} \sum_{k=\ell}^{n-1} s_{k} .
$$

According to John-Nirenberg inequality, sequences of bounded mean oscillation grow at most logarithmically. This agrees well with the Steklov bound (3).

The measure $\mu_{-1}$ in Theorem 1 is the orthogonality measure for the second kind polynomials generated by $\mu$. Given $\mu$, it is possible to construct the measure $\mu_{-1}$ not knowing the recurrence coefficients $\left\{\alpha_{k}\right\}$. Theorem 1 then can be reformulated without referring to $\mu_{-1}$. For more details, see Section 3

The author wishes to thank Stanislav Kupin from University Bordeaux 1 who advised me to search for an analogue of Theorem 1 from [2] in the theory of orthogonal polynomials, inspiring this work.

\section{Proof of Theorem 1}

Let $\mu$ be a probability measure on the unit circle $\mathbb{T}$ supported on a set of infinitely many points, and let $\left\{\Phi_{n}\right\}_{n \geqslant 0}$ be the sequence of monic polynomials orthogonal with respect to $\mu$. Recall that the polynomials $\Phi_{n}$ are determined by relations

$$
\operatorname{deg} \Phi_{n}=n, \quad\left(\Phi_{n}, \Phi_{k}\right)_{L^{2}(\mu)}=0 \text { for } k \neq n, \quad \hat{\Phi}_{n}(n)=1,
$$

and could be obtained via Gram-Schmidt orthogonalization of $\left\{z^{n}\right\}_{n \geqslant 0}$. These polynomials satisfy the system of recurrence relations

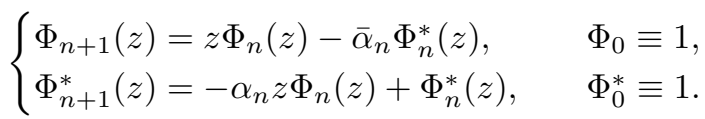

The numbers $\alpha_{n}, n \geqslant 0$, are called the recurrence (or Schur/Verblunski/reflection) coefficients of the measure $\mu$. By definition, we have $\alpha_{n}=-\overline{\Phi_{n+1}(0)}$. For basic theory of orthogonal polynomials on the unit circle we refer the reader to book [9].

Fix a probability measure $\mu$ on the unit circle $\mathbb{T}$ having real recurrence coefficients $\left\{\alpha_{k}\right\}$. Let $\Phi_{n}$ be the monic orthogonal polynomials with respect to $\mu$. For $\alpha \in \mathbb{R}$ define

$$
T(\alpha, z)=\left(\begin{array}{cc}
z & -\alpha \\
-\alpha z & 1
\end{array}\right), \quad T(\alpha)=\left(\begin{array}{cc}
1 & -\alpha \\
-\alpha & 1
\end{array}\right), \quad Q(\alpha)=\left(\begin{array}{cc}
1 & 0 \\
-\alpha & 0
\end{array}\right) .
$$

Then relations (7) yield

$$
\left(\begin{array}{l}
\Phi_{n+1}(z) \\
\Phi_{n+1}^{*}(z)
\end{array}\right)=T\left(\alpha_{n}, z\right) \cdot \ldots \cdot T\left(\alpha_{0}, z\right)\left(\begin{array}{l}
1 \\
1
\end{array}\right), \quad n \geqslant 0 .
$$


In particular, we have

$$
\Phi_{n+1}(1)=\Phi_{n+1}^{*}(1)=\prod_{k=0}^{n}\left(1-\alpha_{k}\right), \quad n \geqslant 0 .
$$

Below in Lemma 2.2 we present a formula in terms of $\left\{\alpha_{k}\right\}$ for derivatives $\Phi_{n+1}^{(j)}(1)$ of order $1 \leqslant j \leqslant n+1$ evaluated at the point 1 . This formula will play a central role in our considerations. For a multi-index $\gamma=\left(\gamma_{0}, \ldots, \gamma_{n}\right)$ of length $n+1$ with components 0 and 1 , put

$$
\Pi(\gamma)=\Pi_{\gamma_{n}}\left(\alpha_{n}\right) \cdot \ldots \cdot \Pi_{\gamma_{1}}\left(\alpha_{1}\right) \Pi_{\gamma_{0}}\left(\alpha_{0}\right),
$$

where $\Pi_{0}(\alpha)=T(\alpha)$ and $\Pi_{1}(\alpha)=Q(\alpha)$ for all $\alpha \in \mathbb{R}$. Denote by $\mathcal{S}_{n, j}$ the set of all multi-indexes $\gamma=\left(\gamma_{0}, \ldots \gamma_{n}\right)$ with components 0,1 such that $\gamma_{0}+\ldots+\gamma_{n}=j$. We start with a simple lemma.

Lemma 2.1. For all integers $n, j$ such that $0 \leqslant j \leqslant n+1$ we have

$$
\left(\begin{array}{c}
\Phi_{n+1}^{(j)}(1) \\
\Phi_{n+1}^{*}(j) \\
(j)
\end{array}\right)=j ! \sum_{\gamma \in \mathcal{S}_{n, j}} \Pi(\gamma)\left(\begin{array}{l}
1 \\
1
\end{array}\right) .
$$

Proof. Formula (9) for $j=0$ and all integers $n \geqslant 0$ is just relation (8) for $z=1$. For $j \geqslant 1$, we can differentiate the expression in formula (8) $j$ times and obtain

$$
\left(\begin{array}{c}
\Phi_{n+1}^{(j)}(1) \\
\Phi_{n+1}^{*(j)}(1)
\end{array}\right)=\left.j ! \sum_{\gamma \in \mathcal{S}_{n, j}} \partial^{\gamma} T\left(\alpha_{n}, z_{n}\right) \cdot \ldots \cdot T\left(\alpha_{0}, z_{0}\right)\right|_{\mathbf{z}}, \quad \partial^{\gamma}=\frac{\partial^{\gamma_{n}}}{\partial z_{n}^{\gamma_{n}}} \cdots \frac{\partial^{\gamma_{0}}}{\partial z_{0}^{\gamma_{0}}},
$$

where $\mathbf{z}=(1,1, \ldots, 1)$ is the vector in $\mathbb{R}^{n+1}$. After substitution $\left.T(\alpha, z)\right|_{z=1}=T(\alpha)$ and $T(\alpha, z)^{\prime} \equiv Q(\alpha)$, we get the desired proposition.

For integers $k_{1}, k_{2}$ and a sequence of real numbers $\left\{c_{k}\right\}$ we denote

$$
\sum_{k=k_{1}}^{k_{2}} c_{k}= \begin{cases}\sum_{k=k_{1}}^{k_{2}-1} c_{k}, & \text { if } k_{1}<k_{2}, \\ 0, & \text { if } k_{1} \geqslant k_{2} .\end{cases}
$$

It will be convenient to put $\alpha_{-1}=0$ and to define the function (sequence) on integers,

$$
h: n \mapsto \prod_{k=-1}^{n-1} \frac{1-\alpha_{k}}{1+\alpha_{k}}, \quad n \geqslant 0 .
$$

Lemma 2.2. For all integers $n, j$ such that $1 \leqslant j \leqslant n+1$, we have

$$
\Phi_{n+1}^{(j)}(1)=\frac{j !}{2^{j}} \prod_{t=0}^{n}\left(1-\alpha_{t}\right) \sum_{t_{1}=0}^{n} \sum_{t_{2}=0}^{t_{1}} \ldots \sum_{t_{j}=0}^{t_{j-1}} \prod_{s=1}^{j}\left(1+\frac{h\left(t_{s}\right)}{h\left(t_{s-1}\right)}\right),
$$

where we put $t_{0}=n+1$.

Proof. Take a multi-index $\gamma \in \mathcal{S}_{n, j}$. Let $0 \leqslant t_{j}<\ldots<t_{1} \leqslant n$ be the indexes such that $\gamma_{t_{s}}=1$ for $1 \leqslant s \leqslant j$. As in the statement of the Lemma, put $t_{0}=n+1$. Using identities

$$
\begin{aligned}
& T(\alpha)\left(\begin{array}{l}
1 \\
1
\end{array}\right)=(1-\alpha)\left(\begin{array}{c}
1 \\
1
\end{array}\right), \quad T(\alpha)\left(\begin{array}{c}
1 \\
-1
\end{array}\right)=(1+\alpha)\left(\begin{array}{c}
1 \\
-1
\end{array}\right), \\
& Q(\alpha)\left(\begin{array}{c}
1 \\
1
\end{array}\right)=Q(\alpha)\left(\begin{array}{c}
1 \\
-1
\end{array}\right)=\frac{1-\alpha}{2}\left(\begin{array}{c}
1 \\
1
\end{array}\right)+\frac{1+\alpha}{2}\left(\begin{array}{c}
1 \\
-1
\end{array}\right),
\end{aligned}
$$


we obtain

$$
\begin{aligned}
\left\langle\Pi(\gamma)\left(\begin{array}{l}
1 \\
1
\end{array}\right)\left(\begin{array}{l}
1 \\
0
\end{array}\right)\right\rangle & =\frac{1}{2^{j}} \prod_{s=1}^{j}\left(\prod_{t=t_{s}}^{t_{s-1}-1}\left(1-\alpha_{t}\right)+\prod_{t=t_{s}}^{t_{s-1}-1}\left(1+\alpha_{t}\right)\right) \cdot \prod_{t=-1}^{t_{j}-1}\left(1-\alpha_{t}\right), \\
& =\frac{1}{2^{j}} \prod_{t=0}^{n}\left(1-\alpha_{t}\right) \cdot \prod_{s=1}^{j}\left(1+\frac{h\left(t_{s}\right)}{h\left(t_{s-1}\right)}\right),
\end{aligned}
$$

Summing up over all multi-indexes $\gamma \in \mathcal{S}_{n, j}$ and using Lemma 2.1, we obtain formula (10).

Denote by $H^{2}(\mu, n)$ the subspace in $L^{2}(\mu)$ consisting of all analytic polynomials of degree at most $n$. Let $k_{\zeta, \mu, n}$ be the reproducing kernel in $H^{2}(\mu, n)$ at $\zeta \in \mathbb{C}$. Define $\pi_{r}=\prod_{k=-1}^{r-1}\left(1-\alpha_{k}^{2}\right)$ for $r \geqslant 0$. The following well-known relation follows from the fact that the family $\left\{\frac{1}{\sqrt{\pi_{r}}} \Phi_{r}\right\}_{0 \leqslant r \leqslant n}$ is the orthonormal basis in $H^{2}(\mu, n)$ :

$$
k_{\zeta, \mu, n}(z)=\sum_{r=0}^{n} \frac{1}{\pi_{r}} \Phi_{r}(z) \overline{\Phi_{r}(\zeta)}, \quad z \in \mathbb{C} .
$$

See Section 2.2 in [9] for more details. For an integer $0 \leqslant j \leqslant n$ we denote by $\bar{\partial}^{j} k_{\xi, \mu, n}$ the derivative of order $j$ of the anti-analytic mapping $\zeta \mapsto k_{\zeta, \mu, n}$ evaluated at a point $\xi \in \mathbb{C}$.

Lemma 2.3. Let $\mu$ be a measure from Steklov class. Then there exists a constant $c$ such that $\left\|\bar{\partial}^{j} k_{1, \mu, n}\right\|_{L^{2}(\mu)}^{2} \leqslant c\left\|\bar{\partial}^{j} k_{1, m, n}\right\|_{L^{2}(m)}^{2}$ for all integers $n \geqslant 0$ and $0 \leqslant j \leqslant n$.

Proof. For every $n \geqslant 0$ consider the operator $T_{\mu, n}$ on $H^{2}(m, n)$ defined by

$$
\left(T_{\mu, n} f, g\right)_{L^{2}(m)}=\int_{\mathbb{T}} f \bar{g} d \mu .
$$

Since the measure $\mu=w d m+\mu_{s}$ belongs to the Steklov class $\mathcal{S}$, we have

$$
\left(T_{\mu, n} f, f\right)_{L^{2}(m)} \geqslant \int_{\mathbb{T}}|f|^{2} w d m \geqslant \inf _{z \in \mathbb{T}} w(z) \cdot\|f\|_{L^{2}(\mathbb{T})}^{2} .
$$

In particular, the operators $T_{\mu, n}, n \geqslant 0$, are invertible and the supremum

$$
c=\sup _{n \geqslant 1}\left\|T_{\mu, n}^{-1}\right\|_{H^{2}(m, n) \rightarrow H^{2}(m, n)}
$$

is finite. Take a point $\zeta \in \mathbb{C}$ and consider $T_{\mu, n}^{-1} k_{\zeta, m, n}$ as an element of $H^{2}(\mu, n)$. For every polynomial $f \in H^{2}(\mu, n)$ we have

$$
\left(f, T_{\mu, n}^{-1} k_{\zeta, m, n}\right)_{H^{2}(\mu, n)}=\left(T_{\mu, n} f, T_{\mu, n}^{-1} k_{\zeta, m, n}\right)_{L^{2}(m)}=f(\zeta)=\left(f, k_{\zeta, \mu, n}\right)_{H^{2}(\mu, n)} .
$$

It follows that $k_{\zeta, \mu, n}=T_{\mu, n}^{-1} k_{\zeta, m, n}$. Differentiating this relation with respect to $\bar{\zeta}$ at $\zeta=1$, we obtain

$$
\bar{\partial}^{j} k_{1, \mu, n}=T_{\mu, n}^{-1} \bar{\partial}^{j} k_{1, m, n}, \quad 0 \leqslant j \leqslant n .
$$

From here and the definition of $c$ we see that $\left\|\bar{\partial}^{j} k_{1, \mu, n}\right\|_{L^{2}(\mu)}^{2} \leqslant c\left\|\bar{\partial}^{j} k_{1, m, n}\right\|_{L^{2}(m)}^{2}$ for all $n$ and $j$, as required.

Lemma 2.4. For all integers $n \geqslant j \geqslant 1$ we have

$$
\left\|\bar{\partial}^{j} k_{1, \mu, n}\right\|_{L^{2}(\mu)}^{2}=\sum_{r=j}^{n} h(r)\left(\frac{j !}{2^{j}} \sum_{t_{1}=0}^{r} \sum_{t_{2}=0}^{t_{1}} \ldots \sum_{t_{j}=0}^{t_{j-1}} \prod_{s=1}^{j}\left(1+\frac{h\left(t_{s}\right)}{h\left(t_{s-1}\right)}\right)\right)^{2},
$$


and $\left\|k_{1, \mu, n}\right\|_{L^{2}(\mu)}^{2}=\sum_{r=0}^{n} h(r)$ for the case where $j=0$.

Proof. For $j=0$ we have

$$
\left\|k_{1, \mu, n}\right\|_{L^{2}(\mu)}^{2}=\sum_{r=0}^{n} \frac{1}{\pi_{r}}\left|\Phi_{r}(1)\right|^{2}=\sum_{r=0}^{n} \frac{1}{\pi_{r}} \prod_{k=-1}^{r-1}\left(1-\alpha_{k}\right)^{2}=\sum_{r=0}^{n} h(r) .
$$

In the case where $j \geqslant 1$ we differentiate (11) and obtain

$$
\left\|\bar{\partial}^{j} k_{1, \mu, n}\right\|_{L^{2}(\mu)}^{2}=\bar{\partial}^{j} k_{1, \mu, n}^{(j)}(1)=\sum_{r=j}^{n} \frac{1}{\pi_{r}}\left|\Phi_{r}^{(j)}(1)\right|^{2} .
$$

Formula (13) now follows readily from formula (10).

For integers $r \geqslant 0, j \geqslant 1$ we define

$$
\kappa_{j}(r)=j ! \sum_{t_{1}=0}^{r} \sum_{t_{2}=0}^{t_{1}} \ldots \sum_{t_{j}=0}^{t_{j-1}} 1= \begin{cases}r ! /(r-j) ! & \text { if } r \geqslant j, \\ 0 & \text { if } r<j .\end{cases}
$$

Lemma 2.5. Let $n, j$ be integer numbers such that $1 \leqslant j \leqslant n / 2$. Denote by $n_{j}$ the integer part of the number $\left(1-\frac{1}{j+1}\right) n$. We have $\frac{\kappa_{j}(n)}{\kappa_{j}\left(n_{j}\right)} \leqslant C$ for a universal constant $C$ independent of $n$ and $j$.

Proof. By Stirling formula, the fraction $\frac{\kappa_{j}(n)}{\kappa_{j}\left(n_{j}\right)}=\frac{n !\left(n_{j}-j\right) !}{n_{j} !(n-j) !}$ is comparable to

$$
\frac{n^{n}\left(n_{j}-j\right)^{n_{j}-j} \sqrt{n\left(n_{j}-j\right)}}{n_{j}^{n_{j}}(n-j)^{n-j} \sqrt{n_{j}(n-j)}} .
$$

We can assume that $n \geqslant 10$. Then for all $1 \leqslant j \leqslant n / 2$ we have

$$
\frac{n}{n_{j}} \leqslant \frac{n}{\left(1-\frac{1}{j+1}\right) n-1} \leqslant \frac{n}{n / 2-1} \leqslant 3, \quad \frac{n_{j}-j}{n-j} \leqslant 1 .
$$

So it suffices to show that the quantity

$$
A_{n, j}=n \log n+\left(n_{j}-j\right) \log \left(n_{j}-j\right)-n_{j} \log n_{j}-(n-j) \log (n-j)
$$

is bounded from above by a constant do not depending on $n \geqslant 10$ and $1 \leqslant j \leqslant n / 2$. For such indexes $n, j$ we have

$$
\frac{n-n_{j}}{n-j} \leqslant \frac{n+1}{(j+1)(n-j)} \leqslant \min \left(\frac{n+1}{2(n-1)}, \frac{n+1}{3(n-n / 2)}\right) \leqslant \frac{3}{4} .
$$

Let $c$ be a constant such that $|\log (1+x)| \leqslant c|x|$ for all $|x| \leqslant \frac{3}{4}$. Then

$$
\begin{aligned}
A_{n, j} & =-n_{j} \log \left(1+\frac{j\left(n-n_{j}\right)}{n\left(n_{j}-j\right)}\right)-j \log \left(1-\frac{n-n_{j}}{n-j}\right)-\left(n-n_{j}\right) \log \left(1-\frac{j}{n}\right) \\
& \leqslant c \frac{j\left(n-n_{j}\right)}{n-j}+c \frac{j\left(n-n_{j}\right)}{n} \leqslant 2 c \frac{j\left(n-n_{j}\right)}{n-j} \leqslant 2 c \frac{j(n+1)}{(j+1)(n-j)} \leqslant 8 c .
\end{aligned}
$$

The lemma follows.

Proof of Theorem 1. We will prove that for every pair of integers $n>\ell \geqslant 0$ one can find an integer $j \leqslant \frac{n}{2}$ depending on $\ell$ such that

$$
\left(\frac{1}{n-\ell} \sum_{k=\ell}^{n-1} e^{2 s_{k}}\right)\left(\frac{1}{n-\ell} \sum_{k=\ell}^{n-1} e^{-2 s_{k}}\right) \leqslant c \frac{\left\|\bar{\partial}^{j} k_{1, \mu, n}\right\|_{L^{2}(\mu)}^{2}\left\|\bar{\partial}^{j} k_{1, \mu_{-1}, n}\right\|_{L^{2}\left(\mu_{-1}\right)}^{2}}{\left\|\bar{\partial}^{j} k_{1, m, n}\right\|_{L^{2}(m)}^{4}},
$$


where $c$ is a universal constant. Since the right hand side is uniformly bounded in $n, j$ by Lemma 2.3, this is sufficient for the proof of the statement.

Let $n, j$ be integer numbers such that $n \geqslant 10$ and $1 \leqslant j \leqslant n / 2$. Denote by $n_{j}$ the integer part of the number $\left(1-\frac{1}{j+1}\right) n$. Using Lemma 2.4 and Jensen's inequality, we obtain

$$
\begin{aligned}
\left\|\bar{\partial}_{1}^{j} k_{1, \mu, n}\right\|_{L^{2}(\mu)}^{2} & =\sum_{r=j}^{n} h(r)\left(\frac{j !}{2^{j}} \sum_{t_{1}=0}^{r} \sum_{t_{2}=0}^{t_{1}} \ldots \sum_{t_{j}=0}^{t_{j-1}} \prod_{s=1}^{j}\left(1+\frac{h\left(t_{s}\right)}{h\left(t_{s-1}\right)}\right)\right)^{2}, \\
& \geqslant \sum_{r=n_{j}}^{n} h(r)\left(\frac{j !}{2^{j}} \sum_{t_{1}=0}^{n_{j}} \sum_{t_{2}=0}^{t_{1}} \ldots \sum_{t_{j}=0}^{t_{j-1}} \prod_{s=1}^{j}\left(1+\frac{h\left(t_{s}\right)}{h\left(t_{s-1}\right)}\right)\right)^{2}, \\
& \geqslant \frac{e^{G_{h, n, j}} \kappa_{j}\left(n_{j}\right)^{2}}{4^{j}} \sum_{r=n_{j}}^{n} h(r),
\end{aligned}
$$

where

$$
G_{h, n, j}=\frac{2}{\kappa_{j}\left(n_{j}\right)} \sum_{t_{1}=0}^{n_{j}} \sum_{t_{2}=0}^{t_{1}} \ldots \sum_{t_{j}=0}^{t_{j-1}} \sum_{s=1}^{j} \log \left(1+\frac{h\left(t_{s}\right)}{h\left(t_{s-1}\right)}\right) .
$$

The same consideration applies to the triple $\mu_{-1},\left\{-\alpha_{k}\right\}, \frac{1}{h}$, and gives

$$
\left\|\bar{\partial}_{1}^{j} k_{1, \mu_{-1}, n}\right\|_{L^{2}\left(\mu_{-1}\right)}^{2} \geqslant \frac{e^{G_{1 / h, n, j}} \kappa_{j}\left(n_{j}\right)^{2}}{4^{j}} \sum_{r=n_{j}}^{n} \frac{1}{h(r)} .
$$

Since $\log (1+a)+\log \left(1+a^{-1}\right) \geqslant \log 4$ for every $a>0$, we see that

$$
\frac{e^{G_{h, n, j}}}{4^{j}} \cdot \frac{e^{G_{1 / h, n, j}}}{4^{j}} \geqslant \frac{e^{2 \sum_{s=1}^{j} \log 4}}{16^{j}}=1 .
$$

It follows that

$$
\left\|\bar{\partial}_{1}^{j} k_{1, \mu, n}\right\|_{L^{2}(\mu)}^{2}\left\|\bar{\partial}_{1}^{j} k_{1, \mu_{-1}, n}\right\|_{L^{2}\left(\mu_{-1}\right)}^{2} \geqslant \kappa_{j}\left(n_{j}\right)^{4}\left(\sum_{r=n_{j}}^{n} h(r)\right)\left(\sum_{r=n_{j}}^{n} \frac{1}{h(r)}\right) .
$$

On the other hand,

$$
\begin{aligned}
\left\|\bar{\partial}_{1}^{j} k_{1, m, n}\right\|_{L^{2}(m)}^{2} & =\sum_{r=j}^{n}\left(j ! \sum_{t_{1}=0}^{r} \sum_{t_{2}=0}^{t_{1}} \ldots \sum_{t_{j}=0}^{t_{j-1}} 1\right)^{2}=\sum_{r=j}^{n}\left(\frac{r !}{(r-j) !}\right)^{2} \\
& =\left(\frac{n !}{(n-j) !}\right)^{2}\left(1+\sum_{r=j}^{n-1}\left(\frac{r-j+1}{r+1} \cdot \ldots \cdot \frac{n-j}{n}\right)^{2}\right) \\
& \leqslant\left(\frac{n !}{(n-j) !}\right)^{2}\left(1+\sum_{r=j}^{n-1}\left(\frac{n-j}{n}\right)^{2(n-r)}\right) \\
& \leqslant\left(\frac{n !}{(n-j) !}\right)^{2} \sum_{s=0}^{\infty}\left(\frac{n-j}{n}\right)^{2 s} \leqslant \frac{n}{j}\left(\frac{n !}{(n-j) !}\right)^{2} .
\end{aligned}
$$


From here we see that

$$
\begin{aligned}
\frac{\left\|\bar{\partial}_{1}^{j} k_{1, \mu, n}\right\|_{L^{2}(\mu)}^{2}\left\|\bar{\partial}_{1}^{j} k_{1, \mu_{-1}, n}\right\|_{L^{2}\left(\mu_{-1}\right)}^{2}}{\left\|\partial_{1}^{j} k_{1, m, n}\right\|_{L^{2}(m)}^{4}} & \geqslant \frac{j^{2} \kappa_{j}\left(n_{j}\right)^{4}}{n^{2} \kappa_{j}(n)^{4}}\left(\sum_{r=n_{j}}^{n} h(r)\right)\left(\sum_{r=n_{j}}^{n} \frac{1}{h(r)}\right) \\
& \geqslant \frac{1}{4 C^{4}}\left(\frac{1}{n-n_{j}} \sum_{r=n_{j}}^{n} h(r)\right)\left(\frac{1}{n-n_{j}} \sum_{r=n_{j}}^{n} \frac{1}{h(r)}\right),
\end{aligned}
$$

where we used Lemma 2.5 and the fact that

$$
\frac{1}{n-n_{j}} \leqslant \frac{1}{n-\left(1-\frac{1}{j+1}\right) n}=\frac{j+1}{n} \leqslant \frac{2 j}{n} .
$$

Now Lemma 2.3 applied to measures $\mu, \mu_{-1}$ from $\mathcal{S}$ yields the inequality

$$
\left(\frac{1}{n-\ell} \sum_{k=\ell}^{n} h(k)\right)\left(\frac{1}{n-\ell} \sum_{k=\ell}^{n} \frac{1}{h(k)}\right) \leqslant 4 C^{4}
$$

for all pairs $n, \ell$ such that $\ell=\left[\left(1-\frac{1}{j+1}\right) n\right]$ for some $1 \leqslant j \leqslant \frac{n}{2}$, where $[a]$ denotes the integer part of a real number $a>0$. By Lemma 2.3 and Lemma 2.4 for $\mu, \mu_{-1}$, and $j=0$, inequality (14) holds in the case where $\ell=0$ as well. Now take arbitrary integers $n>\ell \geqslant 0$ and find maximal integer $j \leqslant \frac{n}{2}$ such that $\ell^{*}=\left[\left(1-\frac{1}{j+1}\right) n\right] \leqslant \ell$. By construction, $n-\ell$ is comparable to $n-\ell^{*}$ with absolute constants. Hence, we can estimate

$$
\frac{1}{(n-\ell)^{2}}\left(\sum_{k=\ell}^{n} h(k)\right)\left(\sum_{k=\ell}^{n} \frac{1}{h(k)}\right) \leqslant \frac{C_{1}}{\left(n-\ell^{*}\right)^{2}}\left(\sum_{k=\ell^{*}}^{n} h(k)\right)\left(\sum_{k=\ell^{*}}^{n} \frac{1}{h(k)}\right) \leqslant C_{2} .
$$

It follows that inequality (14) holds for all $n, \ell$ and some new constant $C$. By Szegö theorem (11) and the definition of Steklov class $\mathcal{S}$, we have $\sum_{k \geqslant 0}\left|\alpha_{k}\right|^{2}<\infty$. In particular, $\sup _{k}\left|\alpha_{k}\right|<1$ and

$$
\left|2 s_{n}+\log h(n)\right| \leqslant \sum_{k=0}^{n}\left|2 \alpha_{k}-\log \frac{1+\alpha_{k}}{1-\alpha_{k}}\right| \leqslant c \sum_{0}^{\infty}\left|\alpha_{k}\right|^{2},
$$

for a constant $c$ do not depending on $n$. Now the discrete Muckenhoupt condition (4) follows from formula (14).

\section{Reformulation of Theorem 1, Negative recurrence coefficients}

Theorem 1 could be reformulated in a way avoiding the usage of the "second kind" measure $\mu_{-1}$. For this we need the definition of the Hilbert transform $\mathrm{Hf}$ of a function $f \in L^{1}(\mathbb{T})$ :

$$
H f(z)=f \frac{f(\xi)}{1-\bar{\xi} z} d m(\xi), \quad z \in \mathbb{T} .
$$

It is known that the principal value integral in formula above converges for almost all $z \in \mathbb{T}$, see Section III.1 in [6].

Theorem $\mathbf{1}^{\prime}$. Let $w \geqslant 0$ be a function on $\mathbb{T}$ such that $w(z)=w(\bar{z})$ for almost all $z$. Assume that $\inf _{\mathbb{T}} w>0$ and $\sup _{\mathbb{T}} w<\infty$. If, moreover, $H w$ is bounded on $\mathbb{T}$, then

$$
\sup _{n>\ell \geqslant 0}\left(\frac{1}{n-\ell} \sum_{k=\ell}^{n-1} e^{2 s_{k}}\right)\left(\frac{1}{n-\ell} \sum_{k=\ell}^{n-1} e^{-2 s_{k}}\right)<\infty,
$$


where $s_{k}=\alpha_{0}+\ldots+\alpha_{k}, k \geqslant 0$, denote the partial sums of recurrence coefficients of $\mu=w d m$.

Both Szegö and Baxter conditions,

$$
\sum_{k \geqslant 0}\left|\alpha_{k}\right|^{2}<\infty, \quad \sum_{k \geqslant 0}\left|\alpha_{k}\right|<\infty
$$

are invariant under the multiplication of the sequence $\left\{\alpha_{k}\right\}$ by a number $\lambda$ of unit modulus. The situation for the Steklov class is completely different.

Proposition 3.1. Let $\mu, \mu_{-1}$ be probability measures on the unit circle $\mathbb{T}$ with recurrence coefficients $\left\{\alpha_{k}\right\},\left\{-\alpha_{k}\right\}$, correspondingly. The following are equivalent:

(a) $\mu \in \mathcal{S}$ and $\mu_{-1} \in \mathcal{S}$,

(b) $\mu=w d m$ for a weight $w$ on $\mathbb{T}$ such that $\inf _{\mathbb{T}} w>0, \sup _{\mathbb{T}} w<\infty$, and the Hilbert transform $H w$ is bounded on $\mathbb{T}$.

It is clear from Proposition 3.1 that Theorem 11 is equivalent to Theorem 1. The only thing to note here is that we have $w(z)=w(\bar{z})$ for a strictly positive weight $w$ on $\mathbb{T}$ and almost all $z \in \mathbb{T}$ if and only if the recurrence coefficients of the measure $\mu=w d m$ are real. The latter follows from the fact that $w$ could be weakly approximated by the sequence of weights $w_{n}=\frac{1}{\left|\Phi_{n}^{*}\right|^{2}}$ (see Theorem 1.7.8 in [9]), which satisfy relations $w_{n}(z)=w_{n}(\bar{z}), z \in \mathbb{T}$.

In the proof of Proposition 3.1 we will use a couple of facts from the theory of harmonic functions. First, a measure $\mu$ on $\mathbb{T}$ has bounded density with respect to the Lebesgue measure $m$ on $\mathbb{T}$ if and only if the harmonic extension of $\mu$ to the open unit disk $\mathbb{D}=\{z \in \mathbb{C}:|z|<1\}$,

$$
\mathcal{P} \mu(z)=\int_{\mathbb{T}} \frac{1-|z|^{2}}{|1-\bar{\xi} z|^{2}} d \mu(\xi), \quad z \in \mathbb{D},
$$

is bounded. Second, let $w \in L^{1}(m)$ and denote by $F$ the analytic function in $\mathbb{D}$ such that $\mathcal{P} \mu(z)=\operatorname{Re} F(z), z \in \mathbb{D}$, where $\mu=w d m$. Then $\operatorname{Im} F$ is bounded in $\mathbb{D}$ if and only if the Hilbert transform of $w$ is bounded on $\mathbb{T}$. For the proof of these facts, see, e.g., Sections I.3 and III.1 in [6].

Proof of Proposition 3.1. Consider the analytic function $F$ in the open unit disk such that its real part $\operatorname{Re} F$ coincides with the harmonic extension of $\mu$,

$$
\operatorname{Re} F(z)=\int_{\mathbb{T}} \frac{1-|z|^{2}}{|1-\bar{\xi} z|^{2}} d \mu(\xi), \quad|z|<1,
$$

and $\operatorname{Im} F(0)=0$. The analytic function $\frac{1}{F}$ has positive real part and equals one at the origin, hence there exists a probability measure $\nu$ on $\mathbb{T}$ such that $\operatorname{Re} \frac{1}{F}$ is the harmonic extension of $\nu$. By Theorem 3.2.14 in [9], the recurrence coefficients of $\nu$ coincide with $\left\{-\alpha_{k}\right\}$, that is, $\nu=\mu_{-1}$. Thus, the harmonic extension of $\mu_{-1}$ to the open unit disk has the form

$$
\operatorname{Re}\left(\frac{1}{F(z)}\right)=\frac{\operatorname{Re} F(z)}{(\operatorname{Re} F(z))^{2}+(\operatorname{Im} F(z))^{2}}, \quad|z|<1 .
$$

Consider the case where $\mu, \mu_{-1}$ belong to the Steklov class. Then Re $\frac{1}{F}$, the harmonic extension of $\mu_{-1}$, is such that $\inf _{|z|<1} \operatorname{Re} \frac{1}{F(z)}>0$, and we see from (16) that $\operatorname{Re} F$ must be bounded in $\mathbb{D}$. We also have $\inf _{|z|<1} \operatorname{Re} F(z)>0$ by (15), hence the 
measure $\mu$ is absolutely continuous, $\mu=w d m$, and $\inf _{\mathbb{T}} w>0$, $\sup _{\mathbb{T}} w<\infty$. Moreover, since both functions $\operatorname{Re} F, \operatorname{Re} \frac{1}{F}$ are separated from zero, the function $\operatorname{Im} F$ is bounded in $\mathbb{D}$, see (16). It follows that the Hilbert transform of $w$ is bounded on the unit circle $\mathbb{T}$.

Now consider a measure $\mu$ as in $(b)$. We obviously have $\mu \in \mathcal{S}$. Let $F$ be the analytic function defined by (15). Then $\operatorname{Re} F$ is bounded in $\mathbb{D}$ and we have $\inf _{z \in \mathbb{D}} \operatorname{Re} F(z)>0$. Since the Hilbert transform of $w$ is bounded on $\mathbb{T}$, the function $\operatorname{Im} F$ is bounded in $\mathbb{D}$. Hence Re $\frac{1}{F}$ is a strictly positive bounded function in $\mathbb{D}$. It follows that $\mu_{-1}$ is an absolutely continuous measure on $\mathbb{T}$ whose density is bounded and separated from zero, in particular, we have $\mu_{-1} \in \mathcal{S}$.

Remark. It is not known to the author if there exists a measure $\mu \in \mathcal{S}$ with real recurrence coefficients $\left\{\alpha_{k}\right\}$ that do not obey the discrete Muckenhoupt condition (4). In a similar situation [2] on the real line the Muckenhoupt condition holds for all measures of the form $\mu=w d x$, where $w$ is a strictly positive bounded weight on $(-\infty, \infty)$.

The following result is known for specialists. We include it for completeness.

Proposition 3.2. Let $\left\{\alpha_{k}\right\}$ be a sequence $(-1,0]$. Then the measure $\mu$ generated by $\left\{\alpha_{k}\right\}$ is in the Steklov class $\mathcal{S}$ if and only if $\sum\left|\alpha_{k}\right|<\infty$.

Proof. If $\sum\left|\alpha_{k}\right|<\infty$, the Baxter theorem applies and yields $\mu \in \mathcal{S}$. Conversely, assume that $\mu$ is a measure on $\mathbb{T}$ whose recurrence coefficients $\alpha_{k}$ lie in $(-1,0]$. By Lemma 2.3 and Lemma 2.4 for $j=0$, we have

$$
\sup _{n \geqslant 0} \frac{1}{n} \sum_{k=0}^{n-1} h(k)<\infty \text {. }
$$

Since $\left\{\alpha_{k}\right\} \subset(-1,0]$, the sequence $\{h(n)\}$ is increasing and we have $h(n) \geqslant 1$ for all $n \geqslant 0$. Hence relation (17) is equivalent to $\sup _{n \geqslant 0} h(n)<\infty$. As at the end of the proof of Theorem 1, we have $\sup _{n \geqslant 0}\left|2 s_{n}+\log h(n)\right|<\infty$. This implies the Baxter condition $\sum_{k \geqslant 0}\left|\alpha_{k}\right|<\infty$.

Example. Proposition 3.2 shows that the sequence $\left\{-\frac{1}{4(k+2)}\right\}_{k \geqslant 0}$ correspond to a measure $\mu \notin \mathcal{S}$. On the other hand, this sequence satisfies Szegö condition $\sum\left|\alpha_{k}\right|^{2}<\infty$, Steklov bound inf $s_{n} \geqslant-\frac{1}{2} \log n+c$, and the discrete Muckenhoupt condition (4). Let us construct another sequence $\left\{\alpha_{k}\right\}$ satisfying the Szegö condition and the Steklov bound for which condition (4) is violated. To do this, fix $\delta>\frac{1}{2}$ and construct a sequence of disjoint intervals $I_{n}=\left[l_{n}-\frac{1}{2}, r_{n}+\frac{1}{2}\right]$ such that $r_{n}>$ $l_{n} \geqslant 1$ are integer numbers, $r_{n}-l_{n}$ is comparable to $l_{n}^{\delta} \log l_{n}$, and the number $\kappa_{n}=r_{n}-l_{n}+1$ of integer points in $I_{n}$ is a multiple of 4 . Take an interval $I_{n}$ and divide it into four equal intervals $I_{n, 1}, \ldots I_{n, 4}$, each containing $\kappa_{n} / 4$ integer points. We enumerate these intervals so that $x \leqslant y$ for all $x \in I_{n, i_{1}}, y \in I_{n, i_{2}}, i_{1}<i_{2}$. For $k$ in the left subinterval $I_{n, 1}$ of $I_{n}$ set $\alpha_{k}=\frac{1}{k^{\delta}}$. For $k \in I_{n, 2}$ we define $\alpha_{k}$ so that the resulting sequence in $I_{n, 1} \cup I_{n, 2}$ is odd with respect to the common (half-integer) point of $I_{n, 1}$ and $I_{n, 2}$. Then define $\alpha_{k}$ on $I_{n, 3} \cup I_{n, 4}$ to obtain the even sequence on $I_{n}$ with respect to the center on $I_{n}$. Note that

$$
\sum_{k \in I_{n, 1} \cup I_{n, 2}} \alpha_{k}=0, \quad \sum_{k \in I_{n, 3} \cup I_{n, 4}} \alpha_{k}=0, \quad \sum_{k \in I_{n}} \alpha_{k}=0, \quad n \geqslant 1 .
$$


Finally, for $k$ not in the union $\cup_{n \geqslant 1} I_{n}$ we set $\alpha_{k}=0$. By construction, the sequence $s_{k}=\alpha_{0}+\ldots+\alpha_{k}$ is odd on each interval $I_{n}$ with respect to its center and $s_{l_{n}-1}=0$, $s_{r_{n}}=0$ for all $n \geqslant 1$. In particular, we have

$$
\langle s\rangle_{I_{n}}=\frac{1}{\left|I_{n}\right|} \sum_{k \in I_{n}} s_{k}=0
$$

for the means of $s=\left\{s_{k}\right\}$. Due to symmetricity, we also have

$$
\frac{1}{\left|I_{n}\right|} \sum_{k \in I_{n}}\left|s_{k}\right|=\frac{1}{\left|I_{n, 1}\right|} \sum_{k \in I_{n, 1}} s_{k}=\frac{1}{\left|I_{n, 1}\right|} \sum_{k \in I_{n, 1}} \sum_{l_{n} \leqslant s \leqslant k} \frac{1}{s^{\delta}},
$$

which is comparable to

$$
\frac{1}{r_{n}-l_{n}} \int_{l_{n}}^{r_{n}} \int_{l_{n}}^{k} \frac{1}{x^{\delta}} d x=\frac{1}{(1-\delta)}\left(\frac{r_{n}^{2-\delta}-l_{n}^{2-\delta}}{\left(r_{n}-l_{n}\right)(2-\delta)}-l_{n}^{1-\delta}\right)
$$

with constants depending only on $\delta$. Our choice of $l_{n}$ and $r_{n}$ allows us to estimate the right hand side of (18) from below by $c_{1}(\delta) \log l_{n}$, where $c_{1}(\delta)$ is a constant depending on $\delta$. Thus, we see that

$$
\sup _{n \geqslant 1} \frac{1}{\left|I_{n}\right|} \sum_{k \in I_{n}}\left|s_{k}-\langle s\rangle_{I_{n}}\right|=\sup _{n \geqslant 1} \frac{1}{\left|I_{n}\right|} \sum_{k \in I_{n}}\left|s_{k}\right| \geqslant c_{1}(\delta) \sup _{n \geqslant 1} \frac{1}{\left|I_{n}\right|} \log l_{n}=+\infty .
$$

Using Jensen's inequality and the fact that $e^{|x|} \leqslant e^{x}+e^{-x}$, we obtain

$$
e^{\frac{2}{\left|I_{n}\right|} \sum_{k \in I_{n}}\left|s_{k}-\langle s\rangle_{I_{n}}\right|} \leqslant 2\left(\frac{1}{\left|I_{n}\right|} \sum_{k \in I_{n}} e^{2 s_{k}}\right)\left(\frac{1}{\left|I_{n}\right|} \sum_{k \in I_{n}} e^{-2 s_{k}}\right) .
$$

From Theorem 1 and (19) we see that one of the measures $\mu, \mu_{-1}$ generated by $\left\{\alpha_{k}\right\},\left\{-\alpha_{k}\right\}$, correspondingly, is not in the Steklov class $\mathcal{S}$. On the other hand, we have $\left|\alpha_{k}\right| \leqslant \frac{c_{2}(\delta)}{k^{\delta}}$ for all $k \geqslant 1$ and a constant $c_{2}(\delta)$, hence $\sum\left|\alpha_{k}\right|^{2}<\infty$. We also have

$$
\left|s_{k}\right| \leqslant \sum_{k \in I_{n, 1}} \frac{1}{k^{\delta}} \leqslant c_{3}(\delta) \log k
$$

for all $k \in I_{n}$ and $s_{k}=0$ for $k \notin \cup_{n \geqslant 1} I_{n}$. Multiplying, if needed, the elements of $\left\{\alpha_{k}\right\}$ by a small constant, we can obtain a new sequence for which the Steklov bound $s_{k} \geqslant-\frac{1}{2} \log k+c$ is satisfied. This ends the construction.

\section{REFERENCES}

[1] A. Aptekarev, S. Denisov, and D. Tulyakov. On a problem by Steklov. Journal of the American Mathematical Society, 29(4):1117-1165, 2016.

[2] R. V. Bessonov. Sampling measures, Muckenhoupt Hamiltonians, and triangular factorization. preprint arXiv:1603.07533, accepted in IMRN, 2016.

[3] S. Denisov. Remark on the formula by rakhmanov and steklov's conjecture. Journal of Approximation Theory, 205:102 - 113, 2016.

[4] S. Denisov and S. Kupin. On the growth of the polynomial entropy integrals for measures in the Szegö class. Advances in Mathematics, 241:18-32, 2013.

[5] S. Denisov and K. Rush. On Schur parameters in Steklov's problem. Journal of Approximation Theory, 215:68-91, 2017.

[6] John B. Garnett. Bounded analytic functions, volume 96 of Pure and Applied Mathematics. Academic Press Inc. [Harcourt Brace Jovanovich Publishers], New York, 1981.

[7] E. A. Rahmanov. On Steklov's conjecture in the theory of orthogonal polynomials. Matem. Sb., 108:581-608, 1979.

[8] E. A. Rahmanov. Estimates of the growth of orthogonal polynomials whose weight is bounded away from zero. Mat. Sb. (N.S.), 114(156)(2):269-298, 335, 1981. 
[9] Barry Simon. Orthogonal Polynomials on the Unit Circle. Part 1: Classical Theory. Colloquium Publications. American Mathematical Society, 2004.

[10] Pavel Kondrat'evich Suetin. V. A. Steklov's problem in the theory of orthogonal polynomials. Itogi Nauki i Tekhniki. Seriya "Matematicheskii Analiz", 15:5-82, 1977.

St.Petersburg State University (29b, 14th Line V.O., 199178, St.Petersburg, Russia) and St.Petersburg Department of Steklov Mathematical Institute of Russian Academy of Science (27, Fontanka, 191023, St.Petersburg, Russia)

E-mail address: bessonov@pdmi.ras.ru 